\title{
Red geodésica GNSS de vínculo entre el datum dinámico SIRGAS y el sistema CR05 de Costa Rica
}

\author{
GNSS Geodetic Network for Linking Between the Dynamic Datum SIRGAS and the \\ CR05 System for Costa Rica
}

\author{
María José Rivas-Guzmán \\ mirg90@hotmail.com \\ Centro Nacional de Procesamiento de Datos GNSS \\ Heredia, Costa Rica \\ Jorge Moya-Zamora \\ jorge.moya.zamora@una.cr \\ Centro Nacional de Procesamiento de Datos GNSS \\ Heredia, Costa Rica
}

Recibido-Received: 5/nov/2015 / Aceptado-Accepted: 28/ene/2016 / Publicado-Published: 31/jul/2016

\begin{abstract}
Resumen
Antes de la aparición de las técnicas de posicionamiento, la mayoría de los países consideraban suficientes la utilización de sistemas de referencia definidos localmente para la georreferenciación de actividades topográficas y cartográficas. Sin embargo, con el avance de la tecnología y, especialmente, los sistemas de posicionamiento, se ve la importancia de emplear un sistema de referencia global. Este proyecto recoge la necesidad de vincular el sistema nacional de referencia Costa Rica (CR05) con las técnicas modernas de procesamiento aplicadas a estaciones GNSS de observación continua, con el fin de recomendar un marco de referencia dinámico. La metodología empleada constó de un procesamiento con el software científico Bernese versión 5.0. El procesamiento consideró las normas establecidas para los centros de procesamiento SIRGAS tanto para el ajuste semanal semilibre como para su vinculación al marco SIRGAS y el análisis de cada solución. Se brindan los resultados de las coordenadas geocéntricas $[\mathrm{X}, \mathrm{Y}, \mathrm{Z}]$ de una red compuesta por 17 estaciones GNSS dentro del territorio nacional, referidas a la época 2012,98 cuyas exactitudes están entre los $0,20 \mathrm{~mm}, 0,15 \mathrm{~mm}$ y $4,4 \mathrm{~mm}$ respectivamente.
\end{abstract}

Palabras claves: SIRGAS, CR05, Bernese, Costa Rica.

\begin{abstract}
Before the appearance of positioning techniques, the use of reference systems, locally defined for the georeferencing in topographic and cartographic activities, was sufficient for most countries. However, with the progress of technology, especially of the positioning systems, the use of a global reference system became vital. In order to provide a dynamic reference frame, this project reflects the need of linking the Costa Rican official reference system (CR05) to modern processing techniques applied to continuous GNSS observation stations. The implemented methodology consisted of a processing carried out with the scientific software Bernese version 5.0. This processing took into account the standards established for SIRGAS Processing Centers for the semifree weekly adjustment, the link to SIRGAS reference frame, and the analysis of each solution. The results given are drawn from the geocentric coordinates [X, Y, Z] of a network made up of 17 GNSS stations within the Costa Rican territory. The coordinates are referred to the epoch 2012.98 and their corresponding accuracies are $0.20 \mathrm{~mm}, 0.15 \mathrm{~mm}$ and $4.4 \mathrm{~mm}$ respectively.
\end{abstract}

Keywords: SIRGAS; CR05; Bernese; Costa Rica. 
Por definición, un sistema de referencia se encuentra materializado por su marco de referencia que consiste en estaciones geodésicas ubicadas en el terreno cuyas coordenadas son conocidas, al igual que la exactitud con que fueron determinadas. Conjuntamente, debe acompañarse la época a que refieren las coordenadas y las velocidades de desplazamiento que presentan en el tiempo para asegurar el mantenimiento del marco de referencia a medida que pasan los años ( $\underline{\text { Rivas, 2015). }}$.

En la actualidad, las diferentes técnicas de posicionamiento satelital han tenido una influencia fundamental en los marcos de referencia geodésicos. La naturaleza de las observaciones de estos sistemas hace que la geodesia aumente su importancia no solo en las actividades topográficas y cartográficas, sino también en los fines científicos sobre los diferentes fenómenos que ocurren en el planeta. Por esto, la mayoría de los países están actualizando sus sistemas de referencia respectivos a un sistema global basado en técnicas modernas. Uno de los aspectos fundamentales de la geodesia moderna es la definición y el mantenimiento del Marco Internacional Terrestre de Referencia (ITRF). La realización de este es de importancia para estudiar las propiedades de la Tierra a nivel global, incluidos los cambios del nivel medio del mar, dinámica de las placas tectónicas, subsidencias regionales, deformaciones en límites de placas, entre otros (Instituto Geográfico Agustín Codazzi ,2014).

El actual marco de referencia nacional denominado como CR05 se encuentra desactualizado. Los vértices fueron vinculados a un marco de referencia ya superado y, además, los puntos de esta red pasiva han sufrido deformaciones producto de diferentes eventos de tipo sísmico, principalmente. Es decir, el sistema oficial CR05 se constituyó por medio de mediciones procesadas dentro del datum ITRF2000; sin embargo, ahora se cuenta con el IGb08 y no se ha hecho la actualización de dicho sistema (Sistema de Referencia Geocéntrico para las Américas, 2014).

El mantenimiento de un marco de referencia a través del tiempo requiere de la cuantificación de los cambios o desplazamientos en las coordenadas, ya sea por efectos tectónicos o geodinámicos, tales como el terremoto ocurrido en setiembre 2012 en Nicoya. Este dejó variaciones considerables en el datum CR05 principalmente en la zona de Guanacaste. Este es un ejemplo claro de la necesidad de establecer un marco geodésico de referencia dinámico, con el propósito de lograr su consistencia, perdurabilidad y precisión en el tiempo; este nuevo marco debe ser accesible mediante el uso de técnicas satelitales para posicionamiento y, a su vez, facilitar los procesos de integración y traslado de la información geoespacial existente.

Se presentan los resultados obtenidos al hacer un procesamiento con el software científico Bernese en su versión 5.0 con el fin de obtener la propuesta de una red geodésica que vincule el datum dinámico SIRGAS y el sistema CR05 como posible solución a la desactualización presentada en el país en el ámbito de marcos de referencia.

\section{Metodología}

Dentro del proyecto se trabajó un periodo de 2 años (2011 y 2012) para efectuar el procesamiento semanal de la red de acuerdo con la estrategia usada por SIRGAS. Las estaciones seleccionadas se pueden clasificar entre las pertenecientes a la red SIRGAS-CON para realizar el vínculo a dicho marco de referencia. Se usó un grupo de 45 estaciones de operación continua GNSS en el territorio nacional. Estas estaciones son administradas por: OVSICORI, AYA, ETCG, IGN y UNAVCO. La ubicación geográfica se muestra en la figura 1. 


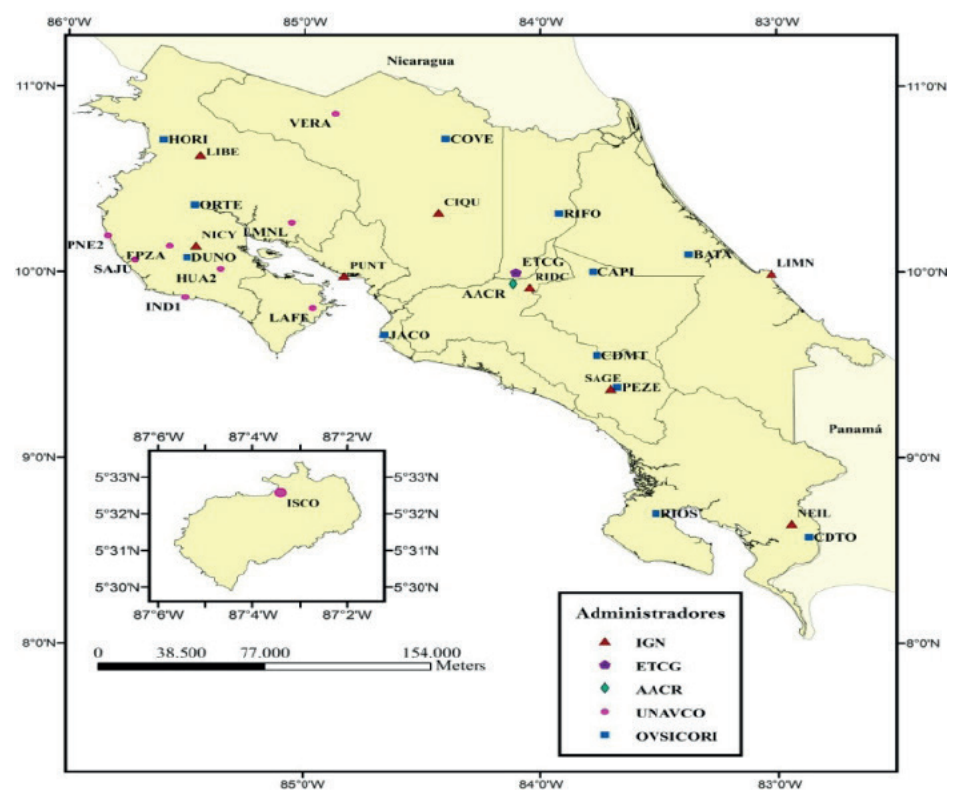

Figura 1. Ubicación de las 45 estaciones GNSS en el territorio nacional. Elaboración propia de la investigación.

Para efectuar el vínculo con el sistema mundial de coordenadas, se emplearon 15 estaciones de medición continua, las cuales forman parte de la red actual SIRGAS-CON y que, a su vez, estas, en su mayoría, deben ser parte del sector de la red calculada por el Centro Nacional de Procesamiento de Datos GNSS (CNPDG) con sede en la Escuela de Topografía, Catastro y Geodesia (ETCG) de la Universidad Nacional en Costa Rica. Las estaciones a utilizar para el amarre al ITRF fueron: AREQ, BOAV, BOGT, CALL, CBSB, CRO1, ETCG, GLPS, GUAT, IGN1, ISCO, MANA, MDO1, RIOP y SCUB, y su ubicación geográfica se muestra en la figura 2.

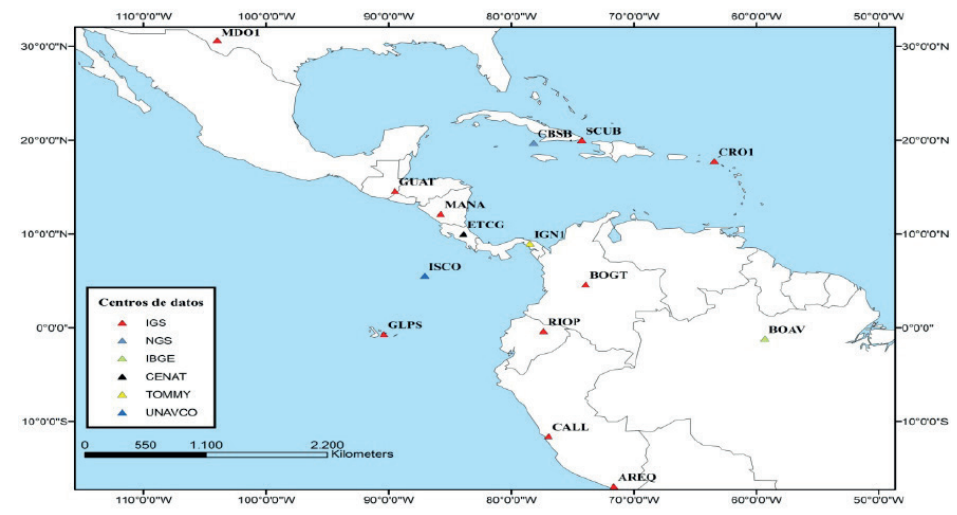

Figura 2. Red de estaciones SIRGAS usadas como vínculo externo. Elaboración propia de la investigación. 
Se usó el software desarrollado por el Instituto Astronómico de la Universidad de Berna - Suiza, denominado Bernese versión 5.0. El Bernese es conocido a nivel internacional por su alto grado de confiabilidad en el procesamiento y ajuste de los datos GPS, debido a la utilización de modelos de corrección para la mayoría de los errores inherentes a las mediciones GPS, proporcionando resultados de exactitud milimétrica (Dach, 2007).

Para el procesamiento de la red se requirió una serie de archivos con diferentes coeficientes y parámetros necesarios para un adecuado cálculo de las observaciones de las estaciones para cada campaña. Estos archivos son facilitados por diferentes entidades mundiales; algunos de ellos están disponibles de manera diaria y otros, por ejemplo, duran hasta dos semanas en ser procesados y estar disponibles. Dentro del procesamiento de cada campaña se deben usar archivos de modelos ionosféricos globales ( ${ }^{*}$.ION), archivos con las efemérides precisas u órbitas finales del IGS ( ${ }^{*}$.SP3), archivos con los parámetros de orientación de la Tierra ( ${ }^{\star}$.IEP), y los archivos de observación de las estaciones de medición continua ( 7 archivos según su disponibilidad). El procesamiento semanal se realiza de forma automática mediante el Bernese Process Engine (BPE), con un archivo empleado por el CNPDG, similar al usado por todos los centros de procesamiento SIRGAS del continente. Una vez configurada la campaña e incorporados todos los archivos necesarios, el procesamiento de cada semana tardó aproximadamente 4,5 horas en ser concluido. La estrategia de procesamiento usada genera soluciones denominadas como semilibres, las cuales consideran como parámetros conocidos las coordenadas de los satélites y los parámetros de orientación terrestre. Este tipo de solución produce una geometría perfecta de la red a través de los vectores entre las estaciones.

Luego de cada procesamiento semanal se procedió a analizar el archivo UNAWWWW7. OUT (las letras W indican el número de la semana GPS) que contiene información general de la campaña, datos estadísticos, las correcciones de las coordenadas y las comparaciones individuales. Dichas comparaciones toman las soluciones diarias con base a la solución combinada para cada semana. Esta comparación se obtiene mediante una transformación entre las soluciones diarias y la combinada, y las tolerancias en las componentes este y norte son $\pm 15 \mathrm{~mm}$ y de $\pm 30 \mathrm{~mm}$ en la vertical.

Los resultados de las campañas semanales permitieron establecer dos criterios para ser usados de filtrado, los cuales son: la repetibilidad y exactitud de las coordenadas.

La repetibilidad proporciona una medida más realista de la exactitud de las coordenadas de la estación y es un indicador de la dispersión de las soluciones de todos los días (Nocquet, 2008). Es decir, la comparación entre la solución semanal combinada y la solución individual. Por lo tanto, este criterio permite cuantificar la magnitud en término de la diferencia y variación de la coordenada de un mismo punto en el transcurso del tiempo. En la tabla 1 se muestra un ejemplo de repetibilidad para la estación ETCG en la semana GPS 1617, el error medio de cada estación se encuentra en la tercera columna y los valores de repetibilidad para cada día de la semana procesada se ubican a partir de la cuarta columna.

Tabla 1

Ejemplo de repetitividad para la estación ETCG, semana 1617

\begin{tabular}{llllllllll}
\hline ETCG & $\mathbf{N}$ & 1.02 & 1.31 & 0.74 & -1.36 & 0.17 & -0.06 & 0.62 & -1.33 \\
ETCG & $\mathbf{E}$ & 1.31 & 0.89 & -2.47 & 0.97 & 1.43 & 0.25 & 0.15 & -0.62 \\
ETCG & $\mathbf{V}$ & 5.27 & 2.45 & -7.94 & 5.18 & 4.49 & 1.22 & 6.42 & -2.75 \\
\hline
\end{tabular}

Nota: Elaboración propia de la investigación. 
ISSN Electrónico: 2215-3470 DOI: http://dx.doi.org/10.15359/ru.30-2.7
UNICIENCIA Vol. 30, No. 2, pp. 87-96. Julio-diciembre, 2016. URL: www.revistas.una.ac.cr/uniciencia Email: revistauniciencia@una.cr

Una vez obtenidas las coordenadas semilibres de la red para las 103 semanas procesadas, se procedió a introducir el marco de referencia, semana por semana, el cual está dado por las coordenadas semanales finales de las estaciones de la red SIRGAS-CON contempladas como amarre. Para la realización del vínculo se emplearon algunos archivos obtenidos en el procesamiento de la red semanalmente:

- Los archivos con las ecuaciones normales diarios de cada semana en formato *.NQ0.

- El archivo semanal con las coordenadas de las estaciones vinculadas al marco SIRGAS y referidas a la época media de cada semana (SIR*PWWWW.CRD).

- Las coordenadas aproximadas de referencia para cada semana (APRWWWW.CRD).

- Las coordenadas semilibres de la combinación semanal (UNAWWWW7.CRD).

Posteriormente, se analizó las diferentes soluciones mediante un módulo interno que permite realizar una transformación de Helmert. Este proceso se hizo comparando y analizando 3 combinaciones de coordenadas mediante la opción Helmert transformation. La primera comparación se llevó a cabo con base en las coordenadas de referencia obtenidas en la vinculación al marco SIRGAS y las coordenadas resultantes. Posteriormente, se comparó la solución semanal semilibre con la solución final, con el fin de poder determinar cuánto es la deformación al momento de introducir el marco de referencia. Por último, se realiza la tercera comparación, que considera las coordenadas semanales finales publicadas por SIRGAS y la solución final.

Con base en lo obtenido de previo, se analizó individualmente el grado de compatibilidad de las soluciones diarias en las coordenadas ajustadas. Se seleccionaron las estaciones de medición continua que cumplían con ciertos criterios para lo que se tomó en cuenta la conformación de una red geodésica de manera homogénea sobre todo el territorio para la conformación de un posible nuevo marco de referencia nacional.

Luego de conformar la nueva propuesta de red GNSS para Costa Rica, se actualizaron los 34 vértices de la red de primer orden desde la época 2005,83 a la época 2011,3 referida al ITRF2005, empleando como insumo las coordenadas ajustadas de 13 vértices y los parámetros de transformación obtenidos de estos mismos mediante Moya et al. (2013).

Finalmente, se transformaron los 21 puntos restantes de la red pasiva al ITRF2005 a la época de referencia 2009,26. Enseguida, se determinaron las coordenadas de las estaciones GNSS del IGN de Costa Rica de la época 2010,35 a la época 2011,3 mediante la aplicación del modelo VEMOS2009 (Drewes, 2009). El uso de este modelo de velocidades es aplicable de manera general en Costa Rica, ya que se ha determinado con los mismos datos generados por la estaciones SIRGAS a través de varias años de observaciones continuas. Luego se calcularon las coordenadas en la época 2011,3. Con las coordenadas calculadas y vinculadas al marco SIRGAS en la época 2011,3 y las coordenadas obtenidas de la aplicación de VEMOS2009, se calculó una transformación de Helmert y sus resultados fueron aplicados a las coordenadas de los puntos de la red pasiva de Primer Orden.

\section{Resultados}

Los resultados del procesamiento permitieron obtener las coordenadas de las estaciones de la red vinculadas al datum SIRGAS; desde la semana GPS 1617 se seleccionaron las estaciones consideradas como óptimas desde los criterios de repetibilidad y exactitud de coordenadas; 
UNICIENCIA Vol. 30, No. 2, pp. 87-96. Julio-diciembre, 2016.

además, se estableció el parámetro de distribución homogénea (mientras que los resultados y los criterios previos lo permitieran). Por otro lado, en la época 2012,68 sucedió un terremoto de magnitud 7,6 Mw a una profundidad de 15,4 Km en la parte occidental de Costa Rica en el sector de Nicoya. Este evento afectó considerablemente algunas de las estaciones de la red geodésica pasiva de Costa Rica, así como algunas de las estaciones GNSS de operación continua (Valverde y Moya, 2013). Si bien es cierto que el terremoto tuvo grandes implicaciones sobre todo en la red pasiva, las estaciones activas tiene una ventaja y es que, precisamente, se puede hacer una cuantificación de estos efectos en sus coordenadas productos de la observación y procesamiento diario (Rivas, 2015). A efectos de dar una solución práctica y válida, en la tabla 2 se brindan las coordenadas geocéntricas finales de los puntos seleccionados de la red GNSS vinculada al marco de referencia SIRGAS, referidas a la época 2012,98 y la cantidad de semanas efectivas en que fue procesada cada estación.

Tabla 2

Coordenadas geocéntricas finales referidas a la época 2012,98 y su cantidad de semanas procesadas

\begin{tabular}{|c|c|c|c|c|}
\hline Estación & $\begin{array}{l}\text { Total de } \\
\text { semanas }\end{array}$ & $\begin{array}{c}X(\mathbf{m}) \\
s_{X}(\mathbf{m m})\end{array}$ & $\begin{array}{c}\mathrm{Y}(\mathbf{m}) \\
\mathbf{s}_{\mathrm{Y}}(\mathbf{m m})\end{array}$ & $\begin{array}{c}\mathrm{Z}(\mathbf{m}) \\
\mathbf{s}_{\mathrm{Z}}(\mathbf{m m})\end{array}$ \\
\hline AACR & 63 & 644008,9980 & $-6251064,2890$ & 1093780,8680 \\
\hline CAPI & 96 & 680978,3716 & $-6247448,3081$ & 1101163,0204 \\
\hline CDMT & 91 & 683680,9224 & $-6256445,6878$ & 1052183,2439 \\
\hline CIQU & 94 & 609048,9247 & $-6246628,6534$ & 1135475,5298 \\
\hline COVE & 69 & 611411,8030 & $-6237766,8986$ & 1178547,0282 \\
\hline EPZA & 64 & 645208,2946 & $-6249842,1569$ & 1100399,5760 \\
\hline ETCG & 96 & 509098,2488 & $-6261454,7217$ & 1102283,2399 \\
\hline HUA2 & 92 & 552167,2400 & $-6261307,4703$ & 1079237,3213 \\
\hline IND1 & 53 & 498678,7127 & $-6249679,7855$ & 1168903,2350 \\
\hline LAFE & 59 & 762717,2534 & $-6235556,5320$ & 1099500,4425 \\
\hline LIBE & 74 & 541235,7947 & $-6253389,5969$ & 1129393,9398 \\
\hline LIMN & 94 & 774610,2419 & $-6258471,5708$ & 952303.0035 \\
\hline LMNL & 97 & 497630,8213 & $-6259516,8919$ & 1115789,5575 \\
\hline NEIL & 94 & 565870,3164 & $-6256745,2049$ & 1098060,8216 \\
\hline NICY & 95 & 651566,5945 & $-6250735,9682$ & 1091707,7467 \\
\hline PUNT & 94 & 690230,7813 & $-6256292,4158$ & 1032020,6174 \\
\hline RIDC & 95 & 469761,2780 & $-6263059,5235$ & 1107572,1045 \\
\hline SAGE & 95 & 644008,9980 & $-6251064,2890$ & 1093780,8680 \\
\hline SAJU & 96 & 680978,3716 & $-6247448,3081$ & 1101163,0204 \\
\hline
\end{tabular}

Nota: Elaboración propia de la investigación. 
Los resultados tales como el promedio de los siete parámetros de transformación de Helmert entre la solución combinada semanal y las soluciones individuales para las 103 semanas procesadas; los parámetros (ver tabla 3) reflejaron que las soluciones individuales solamente sufrieron traslaciones de $1,3 \mathrm{~mm}$ para poder alinearse a la solución con marco, no hubo rotaciones significativas y el valor de la escala es casi nulo. Por otra parte, para los valores de los errores de la repetibilidad de este vínculo, se obtuvo un promedio de - $0,03 \mathrm{~mm}$ para las componentes norte y este, y - $0,45 \mathrm{~mm}$ en el componente de altura.

Tabla 3

Promedio de la transformación de Helmert de 7 parámetros y el error medio

\begin{tabular}{cccc|c|c|c|c|r}
\hline \multirow{2}{*}{ RMS (mm) } & \multicolumn{3}{c|}{ Traslaciones (mm) } & \multicolumn{4}{|c|}{ Rotaciones ( ") } & \multirow{2}{*}{ Escala (ppm) } \\
\cline { 2 - 7 } & $\mathbf{X}$ & $\mathbf{Y}$ & $\mathbf{Z}$ & $\mathbf{X}$ & $\mathbf{Y}$ & $\mathbf{Z}$ & \\
\hline 3,6 & $-0,82$ & 1,77 & 1,56 & $-0,000030$ & $-0,000008$ & 0,000015 & 0,000010 \\
\hline
\end{tabular}

Nota: Elaboración propia de la investigación.

También se analizaron las coordenadas de las soluciones semilibres, las cuales son acordes con el procesamiento, es decir, la consistencia interna de la red tiene una alta exactitud. A su vez, la consistencia externa del marco SIRGAS, dada en la solución SIR11P01, se estableció en un promedio de $\pm 1,0 \mathrm{~mm}$ en las componentes horizontales y en $\pm 2,4 \mathrm{~mm}$ para la componente vertical (SIRGAS, 2014). Por lo tanto, la exactitud final de las coordenadas de los puntos tuvo valores en promedio de unos $\pm 0,20 \mathrm{~mm}, \pm 0,15 \mathrm{~mm}$ y $\pm 4,4 \mathrm{~mm}$ para las coordenadas geocéntricas respectivamente.

Además, se obtuvo un insumo que permitió vincular, de forma aproximada, el sistema oficial CR05 a la nueva red resultante para la época 2011,3 en el ITRF2005. Este insumo se logró mediante la estimación de los 7 parámetros de transformación y sus respectivas exactitudes, las cuales se muestran la tabla 4, las cuales fueron obtenidas entre las coordenadas de la red de estaciones GNSS del IGN determinadas en el proyecto para la época 2011,3 y las coordenadas del IGN actualizadas con VEMOS desde la época 2010,35 hasta a la época 2011,3.

Tabla 4

Parámetros de transformación y sus exactitudes, ITRF2005 época 2011,3

\begin{tabular}{c|c|c|r|r|rr}
\hline \multicolumn{3}{c|}{ Traslaciones (m) } & \multicolumn{3}{c}{ Rotaciones ( ") } \\
\hline $\mathbf{X} / \mathbf{s x}$ & $\mathbf{Y} / \mathbf{s y}$ & $\mathbf{Z} / \mathbf{s z}$ & $\mathbf{X} / \mathbf{s x}$ & \multicolumn{1}{c}{$\mathbf{Y} / \mathbf{s y}$} & \multicolumn{1}{c}{$\mathbf{Z} / \mathbf{s z}$} & Escala (ppm) \\
\hline 0,0150 & 0,0208 & 0,0137 & 0,0024 & $-0,0178$ & 0,0027 & 0,0020 \\
$\pm 0,007$ & $\pm 0,007$ & $\pm 0,007$ & $\pm 0,0333$ & $\pm 0,0121$ & $\pm 0,0207$ & $\pm 0,0583$ \\
\hline
\end{tabular}

Nota: Elaboración propia de la investigación.

Estos parámetros representan las diferencias encontradas entre las coordenadas actualizadas mediante VEMOS y las coordenadas calculadas mediante un software que brinda resultados de alta precisión con respecto a la calidad de las observaciones. Luego de la determinación de los parámetros, se aplicaron estos a la red de primer orden desde la época 2010,35 para obtener como coordenadas resultantes los 34 vértices de la red de primer orden en la época 2011,3 ITRF2005. 
A su vez, se cuantificaron las diferencias existentes entre las determinaciones para los puntos en la época inicial 2005,83, ITRF2000 y la época resultante 2011,3, ITRF 2005. En la figura 4 se representan los valores cuantificados para dar una mejor idea de la situación en cuanto a la ubicación relativa. Con triángulos de color azul se presentan las posiciones oficiales de puntos de la red de primer orden época 2005,83 ITRF 2000, y con los triángulos de color rojo, la posición final calculada. Las líneas azules representan los vectores de desplazamiento de los puntos.

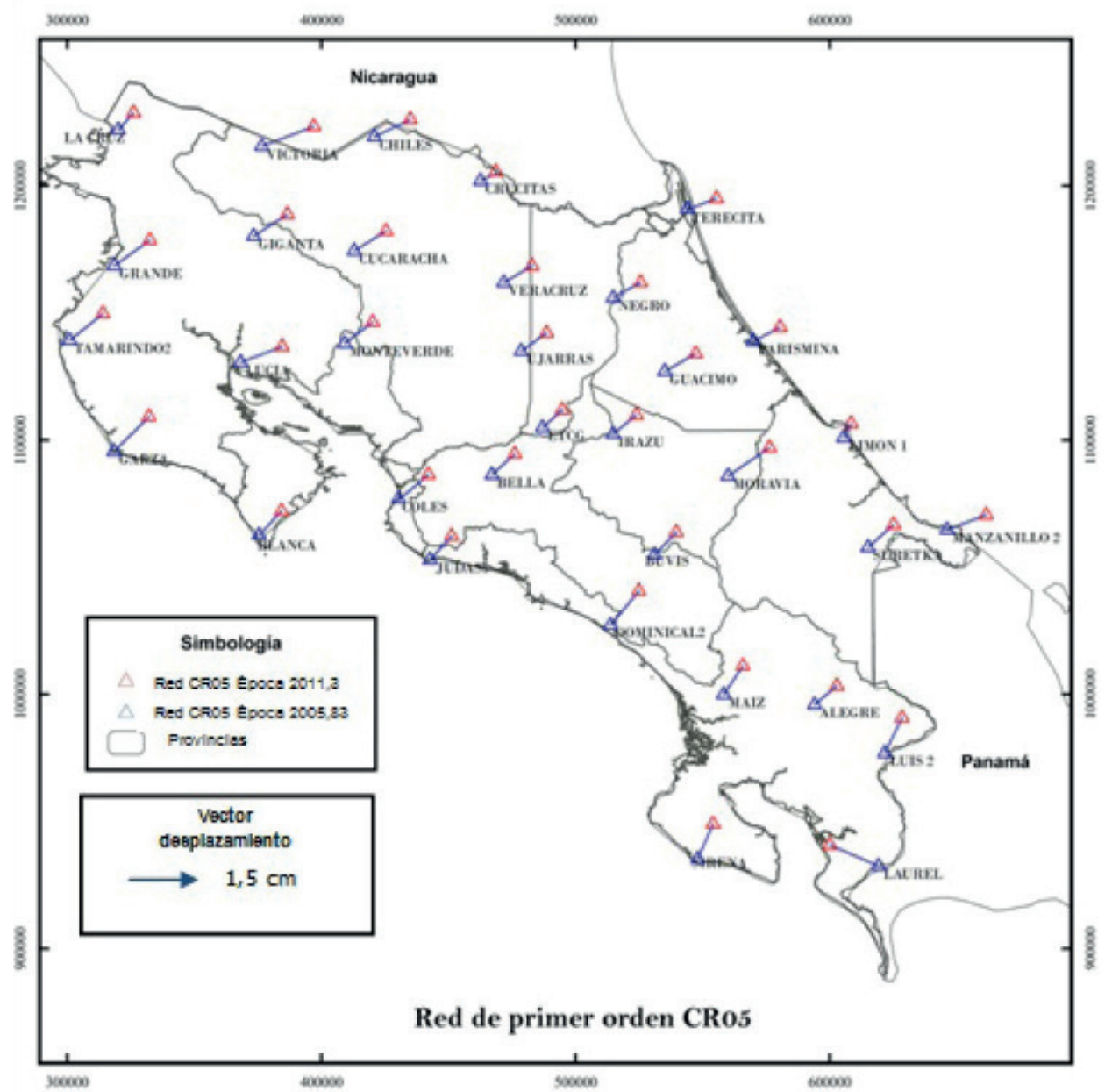

Figura 3. Representación de los desplazamientos entre las épocas 2005,83 y 2011,3. Elaboración propia de la investigación.

Las diferencias entre las coordenadas $\mathrm{X}, \mathrm{Y}, \mathrm{Z}$ obtenidas en la actualización de la red CR05 entre las épocas 2009,26 y 2011,3 muestran así desplazamientos máximos de $6 \mathrm{~cm}, 3 \mathrm{~cm}$ y $5 \mathrm{~cm}$ respectivamente para cada componente. Además, se logró observar un cambio más considerable en la comparación entre las épocas 2005,83 y 2011,30, ya que sus diferencias se encuentran en el orden de los $\pm 10 \mathrm{~cm}$. Sin embargo, hay que considerar que aunque los valores obtenidos están referidos al mismo ITRF por transformación, las soluciones originales provienen de diferentes realizaciones de ITRF, además de que hay una gran influencia de la tectónica de Costa Rica. 
ISSN Electrónico: 2215-3470

DOI: http://dx.doi.org/10.15359/ru.30-2.7
UNICIENCIA Vol. 30, No. 2, pp. 87-96. Julio-diciembre, 2016.

URL: www.revistas.una.ac.cr/uniciencia Email: revistauniciencia@una.cr

Las coordenadas para la época 2011,30 son una estimación realizada y no consideran otros efectos. A su vez, los siete parámetros de transformación determinados en el proyecto ofrecen una aproximación y se encuentran valores significativos en las traslaciones, mientras que el factor de escala es casi nulo y las rotaciones no representan valores significativos.

\section{Conclusiones}

Esta investigación se desarrolló dentro del Centro Nacional de Procesamiento de Datos GNSS de la Escuela de Topografía, Catastro y Geodesia de la Universidad Nacional. Se utilizó la misma estrategia de procesamiento estricto que todos los centros de análisis SIRGAS en todo Latinoamérica y Alemania. Los estándares empleados, los criterios de evaluación y la estrategia de vínculo al marco SIRGAS representan una novedad en el tratamiento, procesamiento y definición de marcos modernos de referencia basados en observaciones GPS.

Se evidencian algunas de las principales diferencias que aventajan un software científico como el Bernese, ya que dicho software permite lograr altas exactitudes en las coordenadas, determinar ciclos perdidos y hacer estimación de velocidades y otra serie de parámetros entre otras posibilidades. A su vez, se destaca una de las principales ventajas la cuál es el uso de modelados para factores como, por ejemplo, el caso de las correcciones a los centros de fase de las antenas, modelados ionosféricos y de carga oceánica. Por el contrario, el procesamiento con software comercial no incluye, en los modelos de procesamiento, la posibilidad de contemplar diferentes modelos y coeficientes que garanticen resultados de alta exactitud.

Los resultados expuestos en este trabajo constituyen un insumo importante en la actualización del datum oficial de Costa Rica, ya que como principal resultado se logró definir coordenadas actualizadas y de alta exactitud en el marco SIRGAS, lo cual es superior a las exactitudes actuales para el marco de referencia CR05.

Se logró obtener como principal resultado una red de alta exactitud conformada por 17 estaciones de operación continua como producto de un procesamiento de aproximadamente 2 años de datos. Las coordenadas geocéntricas de dichas estaciones se encuentran en la época 2012,98 y referidas al datum IGS08, además en dicha red resultante se encuentran 9 estaciones de la red SIRGAS.

La vinculación del sistema CR05 con el sistema propuesto en la época 2011,3 obtuvo diferencias promedio de $\pm 15 \mathrm{~cm}$ en sus coordenadas y en los parámetros de transformación se encontró que tanto las rotaciones como el factor de escala no son significativas. Sin embargo, se debe tener en consideración que los valores establecidos son una buena estimación; pero no son oficiales.

Las diferencias obtenidas del sistema CR05 entre las épocas 2005,83 y 2011,3, confirman el desplazamiento a través del tiempo y, a su vez, puede indicar que el desplazamiento que sufre el territorio nacional de Costa Rica ocurre en una tendencia de movimiento en dirección noreste con el azimut promedio de 40 grados, exceptuando los puntos Limón 1, Laurel y Luis 2, en los cuales se podría plantear la hipótesis de una posible deformación local. 
UNICIENCIA Vol. 30, No. 2, pp. 87-96. Julio-diciembre, 2016.

ISSN Electrónico: 2215-3470

URL: www.revistas.una.ac.cr/uniciencia

DOI: http://dx.doi.org/10.15359/ru.30-2.7

Email: revistauniciencia@una.cr

\section{Referencias}

Dach, R., Hugentobler, U., Fridez, P., Meindl, M. (2007). Bernese GPS Software 5.0. Universidad de Berna. Berna, Suiza.

Drewes, H. (2009). The Actual Kinematic and Crustal Deformation Model APKIM2005 as Basis for NonRotating ITRF. Geodetic Reference Frames, International Association of Geodesy Symposia 134, Vol. 134, pp 95-99.

Instituto Geográfico Agustín Codazzi. (2004). Adopción del marco geocéntrico nacional de referencia MAGNA -SIRGAS como datum oficial de Colombia. Bogotá.

Moya, J. (2013). Parametros de transformacion del sistema de referencia geodésico de Costa Rica CR05ITRF2000 al ITRF2005. Universidad Nacional. Heredia, Costa Rica.

Nocquet, J. M., Mothes, P. y Alvarado, A. (2008). Geodesia, geodinámica y ciclo sísmico en Ecuador. Ecuador.

Rivas, M. (2015). Propuesta de una red geodésica nacional de estaciones de observación continua GNSS como vínculo entre el datum dinámico sirgas y el sistema CR05, mediante el análisis y procesamiento de datos con el software Bernese. Universidad Nacional. Heredia, Costa Rica.

Valverde, J. F. y Moya, J. (2013). Efectos del terremoto del 05 de septiembre de 2012 sobre un conjunto de estaciones GPS de medición continua. Simposio SIRGAS 2013. Ciudad de Panamá, Panamá.

Sistema de Referencia Geocéntrico para las Américas SIRGAS. (2014). SIRGAS. Recuperado de http:// www.sirgas.org.

\section{(c) $(1)(9)$}

Red geodésica GNSS de vínculo entre el datum dinámico SIRGAS y el sistema CR05 de Costa Rica (María José RivasGuzmán y Jorge Moya-Zamora) por Revista Uniciencia se encuentra bajo una Licencia CreativeCommons Atribución-NoComercial-SinDerivadas 3.0 Unported. 